\title{
On $3+1$ decompositions with respect to an observer field via differential forms
}

\author{
Marián Fecko ${ }^{a)}$ \\ Department of Theoretical Physics, Comenius University \\ Mlynská dolina F2, 84215 Bratislava, Slovakia
}

\begin{abstract}
$3+1$ decompositions of differential forms on a Lorentzian manifold $(M, g ;+---)$ with respect to arbitrary observer field and the decomposition of the standard operations acting on them are studied, making use of the ideas of the theory of connections on principal bundles. Simple explicit general formulas are given as well as their application to the Maxwell equations.
\end{abstract}

PCAS : 02.40.-k, 04.20.-q, 03.50.De

running title : On $3+1$ decompositions via differential forms

\section{Introduction}

There is rather extensive literature devoted to $3+1$ split of the laws of physics in curved spacetime (cf. [1] for a review and also the references therein). According to Sec. 2. of [1] there are two rather different methods available to cope with the problem, viz. the congruence method and the hypersurface one.

Here we present a systematic method of $3+1$ split within the congruence method using the language of differential forms on both (4 and $3+1)$ levels.

The use of forms within the $3+1$ decomposition program can be traced back to the classical paper on geometrodynamics [2] (p.581), where it was applied, however, in the framework of the hypersurface method (cf. also [3], pp. 93-94); the time serves there as a parameter labeling the spacelike hypersurfaces and the time derivative of a form is interpreted as a differentiation with respect to a parameter.

The observer field approach similar to ours can be found in [4] (pp. 193-197). What we add here is the introduction of the (simply realized) operator hor (cf. Sec.3) and spatial exterior derivative within the general congruence approach (Sec. 4b). These objects turn out to be very convenient to manipulate with and enable one to derive very simple and at the same time general decomposition formulas and rules.

The following data are assumed in the article : a 4-dimensional Lorentzian ( $g$ of the signature +- - $\left.^{-}\right)$ manifold $M$ with orientation ( $\equiv$ spacetime), and an observer (velocity) field, i.e. a future oriented vector field on $M$ obeying

$$
g(V, V) \equiv\|V\|^{2}=1
$$

(the integral curves of $V$ provide then the congruence of proper-time parametrized worldlines of observers). 
All the constructions are in fact local, i.e. it is enough that the objects mentioned above are available only in a domain $\mathcal{U} \subset M$ rather than globally and consequently no global properties of $M$ are assumed.

\section{The decomposition of forms}

For any vector field $W$ let us define the following standard operations on forms :

$$
\begin{gathered}
i_{W}: \Omega^{p}(M) \rightarrow \Omega^{p-1}(M) \\
j_{W}: \Omega^{p}(M) \rightarrow \Omega^{p+1}(M) \\
i_{W} \alpha(U, \ldots):=\alpha(W, U, \ldots) \\
j_{W} \alpha:=\tilde{W} \wedge \alpha \quad \tilde{W} \equiv g(W, .) \equiv b_{g} W
\end{gathered}
$$

Then the following identity holds :

$$
j_{W} i_{U}+i_{U} j_{W}=g(U, W)
$$

in particular

$$
j_{V} i_{V}+i_{V} j_{V}=1 \equiv \text { identity on } \Omega(M) .
$$

Further, introducing

$$
\mathcal{P}:=i_{V} j_{V} \quad \mathcal{Q}:=j_{V} i_{V}
$$

one checks easily that they represent the set of projection operators on $\Omega^{p}(M)$, i.e.

$$
\mathcal{P}^{2}=\mathcal{P} \quad \mathcal{Q}^{2}=\mathcal{Q} \quad \mathcal{P} \mathcal{Q}=0=\mathcal{Q P} \quad \mathcal{P}+\mathcal{Q}=1
$$

Then for any $\alpha \in \Omega^{p}(M)$ one has

$$
\alpha=(\mathcal{Q}+\mathcal{P}) \alpha=\tilde{V} \wedge i_{V} \alpha+i_{V} j_{V} \alpha
$$

i.e. one obtains the decomposition

$$
\alpha=\tilde{V} \wedge \hat{s}+\hat{r}
$$

where

$$
\hat{s} \equiv i_{V} \alpha \quad \hat{r} \equiv i_{V} j_{V} \alpha
$$

\section{Operator hor and spatial forms}

At any point $m \in M$ we define vertical (instantaneous time) direction - parallel to $V$ and horizontal (instantaneous 3-space) directions - perpendicular to $V$. Then for any vector there is the unique decomposition

$$
U=U_{\|}+U_{\perp} \equiv \operatorname{ver} U+\operatorname{hor} U
$$

and one can define (in the spirit of the theory of connections on principal bundles, cf. [5] )

$$
(\text { hor } \alpha)(U, W, \ldots):=\alpha(\text { hor } U, \text { hor } W, \ldots)
$$

It turns out (cf. Appendix A) that this operation is realized explicitly as

$$
\text { hor } \alpha=i_{V} j_{V} \alpha \equiv \mathcal{P} \alpha \equiv \hat{r}
$$

so that the decomposition in (2.8) can be rewritten also as

$$
\alpha=\tilde{V} \wedge i_{V} \alpha+\text { hor } \alpha
$$

We also introduce the space of purely spatial (horizontal) p-forms by

$$
\hat{\Omega}^{p}(M):=\left\{\alpha \in \Omega^{p}(M) \mid \alpha=\text { hor } \alpha\right\}
$$


(i.e. $\hat{s}=0$ in the decomposition (2.8)) and the Cartan algebra of spatial forms

$$
\hat{\Omega}(M):=\oplus_{p} \hat{\Omega}^{p}(M)
$$

(it is closed with respect to $\wedge$ ). One readily verifies (inserting arguments and using the definition (3.1)) that the projection operator $\mathcal{P} \equiv$ hor is compatible with the algebra structure in $\Omega(M)$

$$
\begin{array}{cc}
\operatorname{hor}(\alpha+\lambda \beta)=\text { hor } \alpha+\lambda \text { hor } \beta & \alpha, \beta \in \Omega(M), \lambda \in \mathbb{R} \\
\text { hor }(\alpha \wedge \beta)=\text { hor } \alpha \wedge \text { hor } \beta & \alpha, \beta \in \Omega(M)
\end{array}
$$

i.e.

$$
\text { hor }: \Omega(M) \rightarrow \operatorname{Im} \text { hor } \equiv \hat{\Omega}(M) \leq \Omega(M)
$$

is an endomorphism of the Cartan algebra $\Omega(M)$. From (3.3) we obtain useful criterion

$$
\alpha=\text { spatial form } \quad \Leftrightarrow \quad i_{V} \alpha=0
$$

Then we see that $\hat{r}, \hat{s}$ in the decomposition (2.8) are spatial ((2.9) plus $\left.i_{V} i_{V}=0\right)$.

Note : If a local orthonormal frame field $e_{a} \equiv\left(e_{0} \equiv V, e_{i}\right)$ and its dual $e^{a} \equiv\left(e^{0} \equiv \tilde{V}, e^{i}\right)$, are used and if

$$
\alpha=\frac{1}{p !} \alpha_{a \ldots b} e^{a} \wedge \ldots \wedge e^{b}
$$

then the decomposition (2.8) is just the split into two parts which do and do not contain respectively the basis 1 -form $e^{0} \equiv \tilde{V}$, i.e.

$$
\alpha=e^{0} \wedge \hat{s}+\hat{r}
$$

where $\hat{s}, \hat{r}$, being spatial, do not already contain the local "time" basis 1-form $e^{0}$, but rather only the "spatial" basis 1 -forms $e^{i}$; explicitly

$$
\hat{s}=\frac{1}{(p-1) !} \alpha_{0 i \ldots j} \underbrace{e^{i} \wedge \ldots \wedge e^{j}}_{p-1} \quad \hat{r}=\frac{1}{p !} \alpha_{k \ldots j} \underbrace{e^{k} \wedge \ldots \wedge e^{j}}_{p}
$$

\section{The decomposition of the operations on forms}

According to (2.8) any form on $(M, g, V)$ can be $3+1$ decomposed as

$$
\alpha=\tilde{V} \wedge \hat{s}+\hat{r}
$$

so that the full information about $\alpha \in \Omega^{p}(M)$ is encoded (in an observer dependent way) into a pair of spatial forms $\hat{s} \in \hat{\Omega}^{p-1}(M)$ and $\hat{r} \in \hat{\Omega}^{p}(M)$. In this section we perform the decomposition of the standard operations on forms, viz. the Hodge star $*$ and the exterior derivative $d$ (some other important operators are then easily obtained by their combinations). By this we mean to introduce some "spatial" operations (acting directly on $\hat{s}, \hat{r}$ and dependent on the observer) producing the same effect as does the given operator acting on $\alpha$.

\section{IVa. The Hodge star}

The horizontal subspace of a tangent space at each point inherits natural metric tensor $\hat{h}$ (with signature + ++ by definition, i.e. $g=\tilde{V} \otimes \tilde{V}-\hat{h}$ ) and orientation (a spatial frame $\left(e_{1}, e_{2}, e_{3}\right)$ is declared to be right-handed if $\left(V \equiv e_{0}, e_{1}, e_{2}, e_{3}\right)$ is right-handed). These data are just enough for the unique spatial Hodge operator

$$
\hat{*}:=*_{\hat{h}}: \hat{\Omega}^{p}(M) \rightarrow \hat{\Omega}^{3-p}(M)
$$

(it is to be applied only on spatial forms). Using the operator

$$
\hat{\eta} \alpha:=(-1)^{\operatorname{deg} \alpha} \alpha
$$


one readily computes (cf. Appendix B) that the decomposition of the "full" Hodge star reads

$$
*(\tilde{V} \wedge \hat{s}+\hat{r})=\tilde{V} \wedge \hat{*} \hat{r}+\hat{*} \hat{\eta} \hat{s}
$$

As an example, applying this to $1 \in \Omega^{0}(M)(\hat{s}=0, \hat{r}=1)$ results in the decomposition of the 4 -volume form

$$
* 1 \equiv \omega=\tilde{V} \wedge \hat{*} 1=: \tilde{V} \wedge \hat{\omega}
$$

where

$$
\hat{\omega}:=\hat{*} 1
$$

is the spatial volume form. In local orthonormal right-handed coframe field $e^{a}$ it is just

$$
\omega \equiv e^{0} \wedge e^{1} \wedge e^{2} \wedge e^{3}=e^{0} \wedge\left(e^{1} \wedge e^{2} \wedge e^{3}\right) \equiv \tilde{V} \wedge \hat{\omega}
$$

\section{IVb. The exterior derivative}

Let $\hat{b}$ be a spatial form, $\mathcal{D}$ a spatial (三 horizontal) domain (i.e. the domain of any possible dimension with the property that any vector tangent to it is horizontal). Then

$$
\begin{aligned}
& \int_{\mathcal{D}} d \hat{b} \stackrel{1 .}{=} \int_{\partial \mathcal{D}} \hat{b} \text { due to Stokes' theorem } \\
& \stackrel{2 .}{=} \int_{\mathcal{D}} \text { hor } d \hat{b} \equiv \int_{\mathcal{D}} \hat{d} \hat{b} \quad \text { since } \mathcal{D} \text { is horizontal } \\
& \qquad \quad \int_{\mathcal{D}} \hat{d} \hat{b}=\int_{\partial \mathcal{D}} \hat{b}
\end{aligned}
$$

where we introduced the spatial exterior derivative

$$
\begin{gathered}
\hat{d}: \hat{\Omega}^{p}(M) \rightarrow \hat{\Omega}^{p+1}(M) \\
\hat{d}:=\text { hor } d \equiv i_{V} j_{V} d
\end{gathered}
$$

(exactly like the covariant exterior derivative of forms on principal bundle with connection). Thus for spatial forms and domains the "full" operator $d$ in the Stokes formula can be replaced by $\hat{d}$. This means that $\hat{d}$ and $\hat{*}$ provide the basic building blocks for the "3-dimensional vector analysis" operations, being the natural generalizations of div, curl etc. used in Minkowski space (div $\sim \hat{*} \hat{d} \hat{*}$, curl $\sim \hat{*} \hat{d}, \ldots)$. We emphasize that the validity of the spatial Stokes formula (4b.1) for $\hat{d}$ is essential for the usefulness and naturality of the latter, e.g. as a means to relate the usual differential $3+1$ laws to the corresponding integral ones (like $\operatorname{div} \mathbf{B}=0 \leftrightarrow$ $\oint \mathbf{B} . d \mathbf{S}=0)$.

So our task now is to express the action of the "full" $d$ operator in terms of $\hat{d}$ (and possibly some other ones) directly on $\hat{s}, \hat{r}$ present in the decomposition (2.8) of $\alpha$. We have

$$
d \alpha=d \tilde{V} \wedge \hat{s}-\tilde{V} \wedge d \hat{s}+d \hat{r}
$$

so that we are to focus our attention to two particular issues, viz. $d$ of $\tilde{V}$ and $d$ of a spatial form.

The decomposition of the 2 -form $d \tilde{V}$ according to (2.8) results in

$$
d \tilde{V}=\tilde{V} \wedge \hat{a}+\hat{y}
$$

with $\hat{a} \in \hat{\Omega}^{1}(M), \hat{y} \in \hat{\Omega}^{2}(M)$. The forms $\hat{a}, \hat{y}$ are the kinematical characteristics of the observer field $V$, which can be easily extracted from any given $V$ using (2.9). Their physical meaning is discussed in Appendix C. It turns out (see also [6], [7], [8]) that $\hat{a}$ equals to the acceleration 1-form

$$
\hat{a}=g\left(\nabla_{V} V, .\right) \equiv g(a, .) \equiv \tilde{a}
$$


( $a \equiv \nabla_{V} V$ is the acceleration field corresponding to $V$ ) and the 2 -form $\hat{y}$, the vorticity form (tensor) is the measure of the (non)integrability of the spatial (horizontal) distribution, i.e. it encodes whether or not the instantaneous 3-spaces mesh together to form a (local) spatial 3-domain $\mathcal{D}$ (or, equivalently, whether or not the time synchronization is possible). These properties of $\hat{a}$ and $\hat{y}$ are reflected in the terminology : $V$ is said to be geodesic if $\hat{a}=0$, irrotational or time-synchronizable if $\hat{y}=0$ and proper-time synchronizable if both $\hat{a}$ and $\hat{y}$ vanish (then $V=\partial_{t}, \tilde{V}=d t$ in adapted coordinates).

The computation of the action of $d$ on a spatial form, as well as on a general form $\alpha$ then, is performed in Appendix D and the result reads

$$
d(\tilde{V} \wedge \hat{s}+\hat{r})=\tilde{V} \wedge\left(-\hat{d} \hat{s}+\mathcal{L}_{V} \hat{r}+\hat{a} \wedge \hat{s}\right)+(\hat{d} \hat{r}+\hat{y} \wedge \hat{s})
$$

The formula (4b.5) gives the desired $3+1$ decomposition of the "full" $d$ operator. Notice the explicit occurrence of both kinematical characteristics $\hat{a}$ and $\hat{y}$.

The spatial exterior derivative $\hat{d}$ shares some important properties with the "full" $d$. In particular, it is the graded derivation of the spatial Cartan algebra $\hat{\Omega}(M)$. Indeed, according to (3.7) we have

$$
\hat{d}(\hat{r} \wedge \hat{R})=\operatorname{hor}(d \hat{r} \wedge \hat{R}+\hat{\eta} \hat{r} \wedge d \hat{R})=\hat{d} \hat{r} \wedge \underbrace{\operatorname{hor} \hat{R}}_{\hat{R}}+\hat{\eta} \underbrace{\operatorname{hor} \hat{r}}_{\hat{r}} \wedge \hat{d} \hat{R}=\hat{d} \hat{r} \wedge \hat{R}+\hat{\eta} \hat{r} \wedge \hat{d} \hat{R}
$$

On the other hand, it is not nilpotent in general, but rather (see Appendices D and G)

$$
\hat{d} \hat{d} \hat{b}=-\hat{y} \wedge \mathcal{L}_{V} \hat{b} \quad \hat{b} \in \hat{\Omega}(M)
$$

holds. This may seem to contradict (4b.1), since the ("full") exterior derivative can be uniquely defined by the ("full") Stokes formula [9] (and it is then nilpotent due to the nilpotence of the boundary operator). The situation can be clarified as follows : for any domain $\mathcal{D}$ and $\alpha \in \Omega(M)$ one has

$$
\int_{\mathcal{D}} d d \alpha=\int_{\partial \partial \mathcal{D}} \alpha=0
$$

( since $\partial \partial=0$ ) which leads to $d d \alpha=0$ identically, i.e. $d$ is nilpotent. For a spatial domain $\mathcal{D}$ and spatial form $\hat{b}$ one has similarly

$$
\int_{\mathcal{D}} \hat{d} \hat{d} \hat{b}=\int_{\partial \partial \mathcal{D}} \hat{b}=0
$$

This does not mean, however, that $\hat{d} \hat{d} \hat{b}=0$ identically, now, but rather $\hat{d} \hat{d} \hat{b}$ should vanish upon restriction to any spatial $\mathcal{D}$. The only nontrivial cases are for the dimension of $\mathcal{D}$ being 3 or 2 . For $\operatorname{dim} \mathcal{D}=3, \hat{y} \neq 0$ (and thus $\hat{d} \hat{d} \hat{b} \neq 0$ due to (4b.7)) means (via Frobenius theorem) that spatial $\mathcal{D}$ (to be used in (4b.9)) does not exist at all. For $\operatorname{dim} \mathcal{D}=2$ we have $\hat{b}=$ function $\equiv f$ and the question is, whether $(V f) \hat{y}$ vanishes (for any $f$ ) upon restriction on any spatial 2-dimensional domain $\mathcal{D}$. This is, however, the case as a result of $\hat{y}$ being the measure of nonintegrability (the bracket of any two vectors tangent to $\mathcal{D}$ is trivially again tangent to $\mathcal{D}$ ). Thus there is no conflict between (4b.1) and (4b.7).

\section{Matrix notation}

For the computation of more complex expressions (e.g. the codifferential in (5.3)) it is quite useful to introduce matrix realization of the operators. If the decomposition (2.8) of $\alpha$ is represented by a column

$$
\alpha \equiv \tilde{V} \wedge \hat{s}+\hat{r} \leftrightarrow\left(\begin{array}{c}
\hat{s} \\
\hat{r}
\end{array}\right)
$$

then e.g.

$$
*(\tilde{V} \wedge \hat{s}+\hat{r})=\tilde{V} \wedge \hat{*} \hat{r}+\hat{*} \hat{\eta} \hat{s} \leftrightarrow\left(\begin{array}{c}
\hat{*} \hat{r} \\
\hat{*} \hat{\eta} \hat{s}
\end{array}\right) \equiv\left(\begin{array}{cc}
0 & \hat{*} \\
\hat{*} \hat{\eta} & 0
\end{array}\right)\left(\begin{array}{c}
\hat{s} \\
\hat{r}
\end{array}\right)
$$

so that

$$
* \leftrightarrow\left(\begin{array}{cc}
0 & \hat{*} \\
\hat{*} \hat{\eta} & 0
\end{array}\right) .
$$


For the exterior derivative we obtain similarly

$$
d(\tilde{V} \wedge \hat{s}+\hat{r}) \leftrightarrow\left(\begin{array}{c}
-\hat{d} \hat{s}+\mathcal{L}_{V} \hat{r}+\hat{a} \wedge \hat{s} \\
\hat{d} \hat{r}+\hat{y} \wedge \hat{s}
\end{array}\right) \equiv\left(\begin{array}{cc}
-\hat{d}+\hat{a} & \mathcal{L}_{V} \\
\hat{y} & \hat{d}
\end{array}\right)\left(\begin{array}{c}
\hat{s} \\
\hat{r}
\end{array}\right)
$$

or

$$
d \leftrightarrow\left(\begin{array}{cc}
-\hat{d}+\hat{a} & \mathcal{L}_{V} \\
\hat{y} & \hat{d}
\end{array}\right)
$$

In the same sense we can then express also other useful operations in terms of such matrices; for the sake of convenience we collect them here together :

$$
\begin{aligned}
& * \leftrightarrow\left(\begin{array}{cc}
0 & \hat{*} \\
\hat{*} \hat{\eta} & 0
\end{array}\right) \quad *^{-1} \leftrightarrow\left(\begin{array}{cc}
0 & -\hat{*} \hat{\eta} \\
\hat{*} & 0
\end{array}\right) \\
& \hat{\eta} \leftrightarrow\left(\begin{array}{cc}
-\hat{\eta} & 0 \\
0 & \hat{\eta}
\end{array}\right) \quad d \leftrightarrow\left(\begin{array}{cc}
-\hat{d}+\hat{a} & \mathcal{L}_{V} \\
\hat{y} & \hat{d}
\end{array}\right) \\
& \delta:=*^{-1} d * \hat{\eta} \leftrightarrow\left(\begin{array}{cc}
0 & -\hat{*} \hat{\eta} \\
\hat{*} & 0
\end{array}\right)\left(\begin{array}{cc}
-\hat{d}+\hat{a} & \mathcal{L}_{V} \\
\hat{y} & \hat{d}
\end{array}\right)\left(\begin{array}{cc}
0 & \hat{*} \\
\hat{*} \hat{\eta} & 0
\end{array}\right)\left(\begin{array}{cc}
-\hat{\eta} & 0 \\
0 & \hat{\eta}
\end{array}\right)= \\
& \left(\begin{array}{cc}
\hat{\delta} & \hat{*}(\hat{y} \wedge \hat{*}) \\
-\hat{*} \mathcal{L}_{V^{*}} & -\hat{\delta}+\hat{*}(\hat{a} \wedge \hat{*} \hat{\eta})
\end{array}\right)
\end{aligned}
$$

where

$$
\hat{\delta}:=\hat{*}^{-1} \hat{d} \hat{*} \hat{\eta}
$$

is the spatial codifferential,

$$
\begin{gathered}
i_{V} \leftrightarrow\left(\begin{array}{ll}
0 & 0 \\
1 & 0
\end{array}\right) \quad j_{V} \leftrightarrow\left(\begin{array}{ll}
0 & 1 \\
0 & 0
\end{array}\right) \\
\text { hor }=i_{V} j_{V} \leftrightarrow\left(\begin{array}{ll}
0 & 0 \\
1 & 0
\end{array}\right)\left(\begin{array}{ll}
0 & 1 \\
0 & 0
\end{array}\right)=\left(\begin{array}{ll}
0 & 0 \\
0 & 1
\end{array}\right) \\
\mathcal{L}_{V} \equiv i_{V} d+d i_{V} \leftrightarrow\left(\begin{array}{cc}
\mathcal{L}_{V} & 0 \\
\hat{a} & \mathcal{L}_{V}
\end{array}\right)
\end{gathered}
$$

\section{The Maxwell equations}

According to the standard conventions on the relationship between the components of the electromagnetic field 2-form $F \equiv \frac{1}{2} F_{a b} e^{a} \wedge e^{b}$ ( $e_{a}$ is $g$-orthonormal frame) and the 3-space vectors of the electric and magnetic fields respectively

$$
F_{0 \alpha}=E_{\alpha}=E^{\alpha} \quad F_{\alpha \beta}=-\epsilon_{\alpha \beta \gamma} B^{\gamma} \equiv-\epsilon_{\alpha \beta \gamma} B_{\gamma}
$$

( $e_{\alpha}$ is $\hat{h}$-orthonormal frame; $\alpha, \beta \ldots$ run from 1 to 3 , being raised and lowered by the spatial metric tensor $\hat{h}_{\alpha \beta}$ $\left.\equiv+\delta_{\alpha \beta} \equiv-\eta_{\alpha \beta}\right)$, one can associate with the electric and magnetic fields the spatial forms

$$
\hat{E}=E_{\alpha} e^{\alpha}=: \mathbf{E} . d \mathbf{r} \quad \hat{B}=B^{\alpha} d S_{\alpha}=: \mathbf{B} . d \mathbf{S} \quad d S_{\alpha}:=\frac{1}{2} \epsilon_{\alpha \beta \gamma} e^{\beta} \wedge e^{\gamma}
$$

Then

$$
F=\tilde{V} \wedge \hat{E}-\hat{B} \leftrightarrow\left(\begin{array}{c}
\hat{E} \\
-\hat{B}
\end{array}\right)
$$

(so that $\hat{s}=\hat{E}, \hat{r}=-\hat{B}$ here). Similarly the electric 4-current 1-form decomposes to

$$
j=j_{a} e^{a}=j_{0} e^{0}+j_{i} e^{i} \equiv \rho \tilde{V}-\hat{j} \leftrightarrow\left(\begin{array}{c}
\rho \\
-\hat{j}
\end{array}\right) \quad \hat{j}:=j_{\alpha} e^{\alpha}=j^{\alpha} e^{\alpha}
$$

Then

$$
* F=\tilde{V} \wedge(-\hat{*} \hat{B})-\hat{*} \hat{E} \leftrightarrow\left(\begin{array}{l}
-\hat{*} \hat{B} \\
-\hat{*} \hat{E}
\end{array}\right)
$$




$$
* j=\tilde{V} \wedge(-\hat{*} \hat{j})+\rho \hat{\omega} \leftrightarrow\left(\begin{array}{c}
-\hat{*} \hat{j} \\
\rho \hat{\omega}
\end{array}\right)
$$

and so the $3+1$ decomposition of the Maxwell equations

$$
\begin{gathered}
d * F=-4 \pi * j \\
d F=0
\end{gathered}
$$

and the continuity equation

$$
d * j=0,
$$

respectively result in

$$
\begin{gathered}
\hat{d} \hat{*} \hat{E}+\hat{y} \wedge \hat{*} \hat{B}=4 \pi \rho \hat{\omega} \\
\hat{d} \hat{*} \hat{B}-\mathcal{L}_{V} \hat{*} \hat{E}-\hat{a} \wedge \hat{*} \hat{B}=4 \pi \hat{*} \hat{j} \\
\hat{d} \hat{E}+\mathcal{L}_{V} \hat{B}-\hat{a} \wedge \hat{E}=0 \\
\hat{d} \hat{B}-\hat{y} \wedge \hat{E}=0
\end{gathered}
$$

and

$$
\mathcal{L}_{V}(\rho \hat{\omega})+\hat{d} \hat{*} \hat{j}-\hat{a} \wedge \hat{*} \hat{j}=0
$$

In particular in the simplest situation, viz. for the irrotational $(\hat{y}=0)$, geodesic $(\hat{a}=0)$ observer field $V($ then $\left.V=\partial_{t}, \tilde{V}=d t\right)$ we get

$$
\begin{gathered}
\hat{d} \hat{*} \hat{E}=4 \pi \rho \hat{\omega} \\
\hat{d} \hat{*} \hat{B}-\mathcal{L}_{\partial_{t}} \hat{*} \hat{E}=4 \pi \hat{*} \hat{j} \\
\hat{d} \hat{E}+\mathcal{L}_{\partial_{t}} \hat{B}=0 \\
\hat{d} \hat{B}=0
\end{gathered}
$$

and

$$
\mathcal{L}_{\partial_{t}}(\rho \hat{\omega})+\hat{d} \hat{*} \hat{j}=0
$$

(there is a simple rule to modify these equations to the case $\hat{a} \neq 0$ but still $\hat{y}=0$; cf. Appendix I).

Since the equations (6.7a) - (6.10) are written in terms of differential forms and standard well-behaved operations with respect to integrals, one can readily write down their corresponding integral versions : let spatial domains of necessary dimensions exist (2-dimensional surface $\mathcal{S}$, 3-dimensional volume $\mathcal{D}$ - the latter case needs $\hat{y}=0$, therefore we put $\hat{y}=0$ in the equations where the integration over 3 -dimensional domain is performed); then

$$
\begin{gathered}
\oint_{\partial \mathcal{D}} \hat{*} \hat{E}=4 \pi \int_{\mathcal{D}} \rho \hat{\omega} \equiv 4 \pi Q \\
\oint_{\partial \mathcal{S}} \hat{*} \hat{B}-\left.\frac{d}{d \tau}\right|_{0} \int_{\Phi_{\tau}(\mathcal{S})} \hat{*} \hat{E}-\int_{\mathcal{S}} \hat{a} \wedge \hat{*} \hat{B}=4 \pi \int_{\mathcal{S}} \hat{*} \hat{j} \\
\oint_{\partial \mathcal{S}} \hat{E}+\left.\frac{d}{d \tau}\right|_{0} \int_{\Phi_{\tau}(\mathcal{S})} \hat{B}-\int_{\mathcal{S}} \hat{a} \wedge \hat{E}=0 \\
\oint_{\partial \mathcal{D}} \hat{B}=0
\end{gathered}
$$

and

$$
\left.\frac{d}{d \tau}\right|_{0} \int_{\Phi_{\tau}(\mathcal{D})} \rho \hat{\omega}+\oint_{\partial \mathcal{D}} \hat{d} \hat{*} \hat{j}-\int_{\mathcal{D}} \hat{a} \wedge \hat{*} \hat{j}=0
$$

where $\Phi_{\tau}$ is the (local) flow generated by $V$.

The equations (6.7a) - (6.10) can be also expressed in more familiar form, making use of 3-dimensional vector analysis operators div, curl etc.; this is done in Appendix H (cf. (H.8) - (H.12)).

Equivalently, if instead of (6.7)

$$
\delta F=4 \pi j
$$


is used, (6.7a), (6.7b) are to be replaced by

$$
\begin{gathered}
\hat{\delta} \hat{E}-\hat{*}(\hat{y} \wedge \hat{*} \hat{B})=4 \pi \rho \\
\hat{\delta} \hat{B}-\hat{*} \mathcal{L}_{V} \hat{*} \hat{E}-\hat{*}(\hat{a} \wedge \hat{*} \hat{B})=4 \pi \hat{j}
\end{gathered}
$$

(they can be obtained directly by applying $\hat{*}$ on $(6.7 \mathrm{a}),(6.7 \mathrm{~b})$, too.)

The decomposition of the 4-potential 1-form

$$
A \leftrightarrow\left(\begin{array}{c}
\phi \\
-\hat{A}
\end{array}\right)
$$

gives

$$
\left(\begin{array}{c}
\hat{E} \\
-\hat{B}
\end{array}\right) \leftrightarrow F \equiv d A \leftrightarrow\left(\begin{array}{cc}
-\hat{d}+\hat{a} & \mathcal{L}_{V} \\
\hat{y} & \hat{d}
\end{array}\right)\left(\begin{array}{c}
\phi \\
-\hat{A}
\end{array}\right)=\left(\begin{array}{c}
-\hat{d} \phi+\phi \hat{a}-\mathcal{L}_{V} \hat{A} \\
\phi \hat{y}-\hat{d} \hat{A}
\end{array}\right)
$$

so that

$$
\begin{gathered}
\hat{E}=-\hat{d} \phi+\phi \hat{a}-\mathcal{L}_{V} \hat{A} \\
\hat{B}=\hat{d} \hat{A}-\phi \hat{y}
\end{gathered}
$$

Finally, the gauge transformation:

$$
A \mapsto A^{\prime} \equiv A+d \chi \leftrightarrow\left(\begin{array}{c}
\phi \\
-\hat{A}
\end{array}\right)+\left(\begin{array}{cc}
-\hat{d}+\hat{a} & \mathcal{L}_{V} \\
\hat{y} & \hat{d}
\end{array}\right)\left(\begin{array}{l}
0 \\
\chi
\end{array}\right)
$$

is

$$
\begin{aligned}
& \phi \mapsto \phi^{\prime} \equiv \phi+V \chi \\
& \hat{A} \mapsto \hat{A}^{\prime} \equiv \hat{A}-\hat{d} \chi
\end{aligned}
$$

\section{Conclusions and summary}

In this article we presented a simple method of $3+1$ decomposition of the physical equations written in terms of differential forms on spacetime $(M, g)$ with respect to a general observer field $V$.

The method consists of the decomposition of both forms and operations on them. The decomposition of forms is based technically on a simple identity (2.4), which can be interpreted in terms of projection operators on $\Omega^{p}(M)$. The decomposition of the operations on forms consists first in the decomposition (4a.2) of the Hodge star operator and then the decomposition of the exterior derivative $d$. Here the formalism mimics the approach used standardly in the theory of connections on principal bundle, viz. we first introduce the operator hor (projecting on the "spatial part" of the form; its simple realization is given by (3.2)) and then define the spatial exterior derivative as $\hat{d}:=$ hor $d$ (the counterpart of the covariant exterior derivative on principal bundle with connection). The decomposition of $d$ is then given by (4b.5). The essential property of $\hat{d}$, which makes it a useful object, is the validity of the spatial Stokes formula (4b.1). It provides the usual link between the differential and integral formulations of the physical laws respectively. The language of differential forms on both 4 and $3+1$ levels turns out to be the most convenient tool for realization of this link, since forms are the objects directly present under the integral signs.

Let us mention, that also the quantities of physical interest which "are not" forms (energy-momentum tensor, Ricci and Einstein tensors,...) admit description in terms of forms [4]; it is then possible to apply the decomposition presented here also to them.

\section{Acknowledgment}

I would like to thank my wife Ľubka for the patience. 
The formula to be proved

$$
(\text { hor } \alpha)(U, \ldots, W) \equiv \alpha(\text { hor } U, \ldots, \text { hor } W)=\left(i_{V} j_{V} \alpha\right)(U, \ldots, W)
$$

is $\mathcal{F}$-linear $\Rightarrow$ it is enough to take either all vector fields $(U, \ldots, W)$ horizontal or one vertical (then $\mathrm{V}$ is enough) and the rest horizontal. The former case means to check

$$
\alpha(U, \ldots, W)=\alpha(U, \ldots, W)-\left(\tilde{V} \wedge i_{V} \alpha\right)(U, \ldots, W),
$$

the latter case

$$
0=\left(i_{V} i_{V} j_{V} \alpha\right)(U, \ldots, W)
$$

Both are easily seen to hold.

\section{Appendix B : Proof of (4a.2)}

In general one has in any orthonormal right-handed frame by definition

$$
* \underbrace{e^{a} \wedge \ldots \wedge e^{b}}_{p}=\frac{1}{(n-p) !} \eta^{a c} \ldots \eta^{b d} \epsilon_{c \ldots d e \ldots f} e^{e} \wedge \ldots \wedge e^{f}
$$

Let

$$
e^{0 i \ldots j}:=e^{0} \wedge \underbrace{e^{i} \wedge \ldots \wedge e^{j}}_{p-1} \quad e^{k \ldots l}:=\underbrace{e^{k} \wedge \ldots \wedge e^{l}}_{p}
$$

be mixed and spatial basis p-forms in $(M^{n} ; g ; \underbrace{+\ldots \ldots-}_{n})$ respectively and let us treat the orthogonal complement to $V \equiv e_{0}$ as the Euclidean space with the signature $(+\ldots+)$ (its dimension is $\left.n-1\right)$. Then

$$
\begin{gathered}
* e^{0 i \ldots j}=\frac{1}{(n-p) !} \underbrace{\eta^{00} \eta^{i i} \ldots \eta^{j j}}_{(-1)^{p-1}} \underbrace{\epsilon_{0 i \ldots j k \ldots l} e^{k \ldots l}}_{((n-1)-(p-1)) ! \hat{*} e^{i \ldots j}}= \\
=(-1)^{p-1} \hat{*} e^{i \ldots j}=\hat{*} \hat{\eta} e^{i \ldots j}
\end{gathered}
$$

and

$$
\begin{gathered}
* e^{k \ldots l}=\frac{1}{(n-p) !} \underbrace{\eta^{k k} \ldots \eta^{l l}}_{(-1)^{p}} \varepsilon_{k \ldots l a \ldots b} e^{a \ldots b}= \\
=\frac{(-1)^{p}}{(n-p) !}(n-p) \varepsilon_{k \ldots l 0 r \ldots s} e^{0 r \ldots s}= \\
=\frac{1}{(n-p-1) !} \varepsilon_{0 k \ldots l r \ldots s} e^{0 r \ldots s}=e^{0} \wedge \hat{*} e^{k \ldots l}
\end{gathered}
$$

Then if

$$
\begin{array}{rl}
\hat{s} \equiv \frac{1}{(p-1) !} \hat{s}_{i \ldots j} e^{i \ldots j} & (p-1)-\text { form } \\
\hat{r} \equiv \frac{1}{p !} \hat{r}_{k \ldots l} e^{k \ldots l} & p-\text { form }
\end{array}
$$

and

$$
\alpha=e^{0} \wedge \hat{s}+\hat{r}
$$

we have

$$
*\left(e^{0} \wedge \hat{s}+\hat{r}\right)=\frac{1}{(p-1) !} \hat{s}_{i \ldots j} * e^{0 i \ldots j}+\frac{1}{p !} \hat{r}_{k \ldots l} * e^{k \ldots l}=e^{0} \wedge \hat{*} \hat{r}+\hat{*} \hat{\eta} \hat{s}
$$


Let

$$
d \tilde{V}=\tilde{V} \wedge \hat{a}+\hat{y}
$$

be the decomposition (4b.3) of the 2 -form $d \tilde{V}$.Then according to (2.9) one has

$$
\begin{gathered}
\hat{a}=i_{V} d \tilde{V}=(\underbrace{i_{V} d+d i_{V}}_{\mathcal{L}_{V}}) \tilde{V}-d \underbrace{i_{V} \tilde{V}}_{\|V\|^{2}=1}=\mathcal{L}_{V} \tilde{V}= \\
=\mathcal{L}_{V}(g(V, .))=\left(\mathcal{L}_{V} g\right)(V, .)+g(\underbrace{\mathcal{L}_{V} V}_{[V, V]=0}, .)=\left(\mathcal{L}_{V} g\right)(V, .)
\end{gathered}
$$

However, for the Levi-Civita connection one has in arbitrary coordinates

$$
\begin{gathered}
\left(\mathcal{L}_{V} g\right)_{i j}=V_{i ; j}+V_{j ; i} \\
\Rightarrow \quad\left(\left(\mathcal{L}_{V} g\right)(V, .)\right)_{i}=V^{j} V_{i ; j}+V^{j} V_{j ; i}=\left(\nabla_{V} \tilde{V}\right)_{i}+(d \frac{1}{2} \underbrace{\|V\|^{2}}_{1})_{i}
\end{gathered}
$$

so that

$$
\hat{a}=\left(\mathcal{L}_{V} g\right)(V, .)=\nabla_{V} \tilde{V}=\underbrace{\left(\nabla_{V} g\right)}_{0}(V, .)+g\left(\nabla_{V} V, .\right) \equiv g(a, .)
$$

where

$$
a:=\nabla_{V} V
$$

is the acceleration field corresponding to the observer field $V$; thus

$$
\hat{a}=g(a, .) \equiv \tilde{a}
$$

The 2-form $\hat{y}$ :

According to the Frobenius theorem, $\hat{y} \neq 0$ means the nonintegrability of the horizontal (3-space) distribution. This can be rephrased as the impossibility of the synchronization of the clocks within the 3-space for the observers moving along $V$, too. Indeed, let

$$
t: \mathcal{U} \rightarrow \mathbb{R}
$$

be a time function (coordinate) in a 4-region $\mathcal{U}$, synchronized for any two nearby space-related points, i.e.

1. $W t=0$ for any horizontal $W$ ( $t$ is constant along the instantaneous 3 -space)

2. $V t \equiv \chi>0$ ( time increases along any observer's worldline).

The condition 1 . can be rewritten also as

$$
0=(\text { hor } W) t=\langle d t, \text { hor } W\rangle=\langle\text { hor } d t, W\rangle \equiv\langle\hat{d} t, W\rangle
$$

for any $W$, i.e.

$$
\hat{d} t=0
$$

as a 1-form. According to (D.1)

$$
0=\hat{d} t=d t-(V t) \tilde{V} \equiv d t-\chi \tilde{V}
$$

or

$$
\tilde{V}=\psi d t \quad \psi \equiv \chi^{-1}>0
$$

Then

$$
\begin{gathered}
d \tilde{V}=d \psi \wedge d t=\frac{d \psi}{\psi} \wedge \psi d t=\tilde{V} \wedge\left(-\frac{d \psi}{\psi}\right)= \\
=\tilde{V} \wedge\left(-\frac{\hat{d} \psi+(V \psi) \tilde{V}}{\psi}\right)=\tilde{V} \wedge\left(-\frac{\hat{d} \psi}{\psi}\right)
\end{gathered}
$$

Comparison with (4b.3) then gives

$$
\hat{y}=0 \quad \hat{a}=-\frac{\hat{d} \psi}{\psi} \equiv-\hat{d} \Phi \quad \psi \equiv e^{\Phi}
$$


Thus $\hat{y} \neq 0$ is the obstacle for existence of a time function $t$ synchronized in 3-space and (if $\hat{y}=0$ ) $\Phi:=\ln \psi$ is the "gravitational potential" ([6], p.33).

\section{Appendix D : The proofs of (4b.5), and (4b.7).}

Let $\hat{b}$ be any spatial form, i.e. $i_{V} \hat{b}=0$; then

$$
\begin{gathered}
d \hat{b}=\left(j_{V} i_{V}+i_{V} j_{V}\right) d \hat{b}=\tilde{V} \wedge \underbrace{i_{V} d}_{\mathcal{L}_{V}-d i_{V}} \hat{b}+\underbrace{i_{V} j_{V} d}_{\hat{d}} \hat{b}= \\
=\tilde{V} \wedge \mathcal{L}_{V} \hat{b}+\hat{d} \hat{b}-\tilde{V} \wedge d \underbrace{i_{V} \hat{b}}_{0}
\end{gathered}
$$

so that on spatial forms

$$
d \hat{b}=\tilde{V} \wedge \mathcal{L}_{V} \hat{b}+\hat{d} \hat{b}
$$

Then on a general form $\alpha$

$$
\begin{gathered}
d \alpha=d(\tilde{V} \wedge \hat{s}+\hat{r})=d \tilde{V} \wedge \hat{s}-\tilde{V} \wedge d \hat{s}+d \hat{r}= \\
=(\tilde{V} \wedge \hat{a}+\hat{y}) \wedge \hat{s}-\tilde{V} \wedge\left(\tilde{V} \wedge \mathcal{L}_{V} \hat{s}+\hat{d} \hat{s}\right)+\tilde{V} \wedge \mathcal{L}_{V} \hat{r}+\hat{d} \hat{r}= \\
=\tilde{V} \wedge\left(\mathcal{L}_{V} \hat{r}-\hat{d} \hat{s}+\hat{a} \wedge \hat{s}\right)+(\hat{d} \hat{r}+\hat{y} \wedge \hat{s})
\end{gathered}
$$

where all forms in the brackets are already spatial.

The computation of $\hat{d} \hat{d}$ : for arbitrary horizontal form $\hat{b}$

$$
\begin{gathered}
\hat{d} \hat{d} \hat{b} \stackrel{(D .1)}{=} \operatorname{hor} d\left(d \hat{b}-\tilde{V} \wedge \mathcal{L}_{V} \hat{b}\right)=-\operatorname{hor}\left(d \tilde{V} \wedge \mathcal{L}_{V} \hat{b}-\tilde{V} \wedge d \mathcal{L}_{V} \hat{b}\right) \stackrel{(3.7)}{=} \\
=-\underbrace{\operatorname{hor} d \tilde{V}}_{\hat{y}} \wedge \underbrace{\operatorname{hor} \mathcal{L}_{V} \hat{b}}_{\mathcal{L}_{V} \hat{b}}+\underbrace{\operatorname{hor} \tilde{V}}_{0} \wedge \operatorname{hor} d \mathcal{L}_{V} \hat{b}=-\hat{y} \wedge \mathcal{L}_{V} \hat{b}
\end{gathered}
$$

since

$$
\text { hor } \mathcal{L}_{V} \hat{b}=i_{V} j_{V}(i_{V} d+d \underbrace{\left.i_{V}\right) \hat{b}}_{0}=i_{V} \underbrace{j_{V} i_{V}}_{1-i_{V} j_{V}} d \hat{b}=i_{V} d \hat{b}-\underbrace{i_{V} i_{V}}_{0} j_{V} d \hat{b}=(i_{V} d+d \underbrace{\left.i_{V}\right) \hat{b}}_{0}=\mathcal{L}_{V} \hat{b}
$$

\section{Appendix E : The volume expansion coefficient $\theta$}

According to (4a.2)

$$
\omega \equiv * 1=\tilde{V} \wedge \hat{*} 1 \equiv \tilde{V} \wedge \hat{\omega}
$$

where $\hat{\omega} \equiv \hat{*} 1$ is the spatial volume form. The standard definition of the volume expansion coefficient $\theta$ is ([6], p. 9)

$$
\theta:=V_{; \mu}^{\mu} \equiv \nabla \cdot V \equiv \operatorname{div} V
$$

Since

$$
\mathcal{L}_{V} \omega=(\operatorname{div} V) \omega \equiv \theta \omega
$$

one can write

$$
\begin{aligned}
\theta \omega=\tilde{V} \wedge(\theta \hat{\omega})=\mathcal{L}_{V} \omega & =\left(\mathcal{L}_{V} \tilde{V}\right) \wedge \hat{\omega}+\tilde{V} \wedge \mathcal{L}_{V} \hat{\omega}=(\text { cf. App.C }) \\
& =\hat{a} \wedge \hat{\omega}+\tilde{V} \wedge \mathcal{L}_{V} \hat{\omega}
\end{aligned}
$$

The first term vanishes (spatial 4-form), $\mathcal{L}_{V} \hat{\omega}$ is spatial (the end of App.D) so that

$$
\mathcal{L}_{V} \hat{\omega}=\theta \hat{\omega}
$$


which means that $\theta$ is the rate of change of 3-volumes along the observer's wordline $\gamma($ with $\dot{\gamma}=V$ ). As an example $\mathcal{L}_{V}(\rho \hat{\omega})=(V \rho+\theta) \hat{\omega}($ see $(6.10))$.

\section{Appendix $\mathbf{F}$ : The identities resulting from $d d=0$}

Applying $d$ on the decomposition (4b.3) and using (4b.5) one obtains

$$
\begin{aligned}
0 & =d d \tilde{V}=d \tilde{V} \wedge \hat{a}-\tilde{V} \wedge d \hat{a}+d \hat{y}= \\
& =\tilde{V} \wedge\left(\mathcal{L}_{V} \hat{y}-\hat{d} \hat{a}\right)+(\hat{d} \hat{y}+\hat{y} \wedge \hat{a})
\end{aligned}
$$

so that $\hat{a}$ and $\hat{y}$ are always related by

$$
\begin{gathered}
\hat{d} \hat{a}=\mathcal{L}_{V} \hat{y} \\
\hat{d} \hat{y}=-\hat{y} \wedge \hat{a}
\end{gathered}
$$

In particular, for $\hat{y}=0$ we get

$$
\hat{d} \hat{a}=0
$$

From (C.8) we even know, that

$$
\hat{a}=-\hat{d} \Phi
$$

in this case.

Applying $d d=0$ on a spatial form $\hat{r}$ and taking into account (F.1), (F.2) we obtain another useful identity

$$
\left[\mathcal{L}_{V}, \hat{d}\right]=-\hat{a} \wedge \mathcal{L}_{V} \quad \text { on } \hat{\Omega}(M)
$$

(this shows that "time" derivative $\mathcal{L}_{V}$ and "space" derivatives hidden in $\hat{d}$ do not commute in general; they do commute, however, for the geodesic observer field $(\hat{a}=0)$ ).

\section{Appendix G : Formal links with the theory of connections on principal bundles}

The formulation used in this article resembles in many respects the theory of connections on principal bundles [5]. There is a (right) action $R_{g}$ of a Lie group $\mathrm{G}$ on the total space $P$, if we set $G \equiv(\mathbb{R},+$ ), $P \equiv M$ (globally one needs $V$ to be complete for this) and the action is identified with the flow generated by $V$. The difference is, however, that the horizontal distribution here is not $G$-invariant in general : since $\tilde{V}$ is the counterpart of the connection form $\omega$ - both define the horizontal distribution via annihilation - and the group is 1-dimensional, the $(\mathbb{R},+)$ - invariance means $\mathcal{L}_{V} \tilde{V}=0$. But

$$
\mathcal{L}_{V} \tilde{V} \stackrel{(C .3)}{=} \hat{a} \neq 0 \quad \text { in general }
$$

(thus for geodesic (三nonaccelerating) observer field there $i s$ in fact $\mathbb{R}$-connection available).

Many formulas here are very similar to those in the connection theory; e.g.

$$
\hat{d} \hat{b}=d \hat{b}-\tilde{V} \wedge \mathcal{L}_{V} \hat{b}
$$

(cf. (D.1)) is the counterpart of the standard formula valid for the computation of the covariant exterior derivative of the horizontal form $\alpha$ of type $\rho$, viz.

$$
D \alpha=d \alpha+\rho^{\prime}(\omega) \dot{\wedge} \alpha
$$

where $\omega$ is the connection form, $\rho$ a representation of $G$. To see this more explicitly, one has to notice that the forms of type $\rho$ satisfy

$$
\mathcal{L}_{\xi_{i}} \alpha=-\rho^{\prime}\left(E_{i}\right) \alpha
$$

( $\xi_{i} \equiv \xi_{E_{i}}$ being the fundamental field corresponding to the basis element $E_{i}$ of the Lie algebra $\mathcal{G}$ of $G$ ) and consequently

$$
\rho^{\prime}(\omega) \dot{\wedge} \alpha=\omega^{i} \wedge \rho^{\prime}\left(E_{i}\right) \alpha=-\omega^{i} \wedge \mathcal{L}_{\xi_{i}} \alpha
$$


Or

$$
D \alpha=d \alpha-\omega^{i} \wedge \mathcal{L}_{\xi_{i}} \alpha
$$

This formula is valid for all horizontal forms on the principal bundle (not only of type $\rho$ ). For 1-dimensional group (as is the case here) we have exactly the form (G.2).

In the same way one can see the similarity of

$$
\hat{d} \hat{d} \hat{b}=-\hat{y} \wedge \mathcal{L}_{V} \hat{b}
$$

(4b.7) with the standard formula

$$
D D \alpha=\rho^{\prime}(\Omega) \dot{\wedge} \alpha \equiv \Omega^{i} \wedge \rho^{\prime}\left(E_{i}\right) \alpha
$$

where $\Omega$, the curvature 2 -form, is the counterpart of our $\hat{y}$ : both encode the (non)integrability of the horizontal distribution and consequently both are computed by the same rule, viz.

$$
\hat{y}:=\operatorname{hor} d \tilde{V} \equiv \hat{d} \tilde{V} \quad \text { versus } \quad \Omega:=\text { hor } d \omega \equiv D \omega .
$$

The counterpart of the Bianchi identity DD $\omega=0$ is

$$
\hat{d} \hat{d} \tilde{V}=\hat{d} \hat{y} \stackrel{(F .2)}{=}-\hat{y} \wedge \hat{a}
$$

This is not zero in general, but it is zero for $\hat{a}=0$, where $\tilde{V}$ does define a connection.

\section{Appendix H : The Maxwell equations in the standard vector analysis notations}

We use the standard 3-dimensional Euclidean space relations (cf. definitions in (6.2))

$$
\begin{gathered}
\hat{E}=\mathbf{E} \cdot d \mathbf{r} \quad \hat{B}=\mathbf{B} \cdot d \mathbf{S} \quad \hat{j}=\mathbf{j} \cdot d \mathbf{r} \quad \hat{y}=\mathbf{y} \cdot d \mathbf{S} \quad \hat{a}=\mathbf{a} \cdot d \mathbf{r} \\
\hat{*} \hat{E}=\mathbf{E} \cdot d \mathbf{S} \quad \hat{*} \hat{B}=\mathbf{B} \cdot d \mathbf{r} \quad \hat{*} \hat{j}=\mathbf{j} \cdot d \mathbf{S} \quad \hat{*} \hat{y}=\mathbf{y} \cdot d \mathbf{r} \quad \hat{*} \hat{a}=\mathbf{a} \cdot d \mathbf{S} \\
\hat{y} \wedge \hat{*} \hat{B}=(\mathbf{y} \cdot \mathbf{B}) \hat{\omega} \quad \hat{a} \wedge \hat{*} \hat{B}=(\mathbf{a} \times \mathbf{B}) \cdot d \mathbf{S} \quad \hat{a} \wedge \hat{*} \hat{j}=(\mathbf{a} \cdot \mathbf{j}) \hat{\omega} \\
\hat{y} \wedge \hat{E}=(\mathbf{y} \cdot \mathbf{E}) \hat{\omega} \quad \hat{a} \wedge \hat{E}=(\mathbf{a} \times \mathbf{E}) \cdot d \mathbf{S}
\end{gathered}
$$

One can then introduce curl and div operations according to

$$
\begin{aligned}
& \hat{d} \hat{E}=:(\operatorname{curl} \mathbf{E}) \cdot d \mathbf{S} \\
& \hat{d} \hat{*} \hat{E}=:(\operatorname{div} \mathbf{E}) \hat{\omega}
\end{aligned}
$$

and consequently then also

$$
\begin{gathered}
\hat{d} \hat{B}=:(\operatorname{div} \mathbf{B}) \hat{\omega} \\
\hat{d \hat{*} \hat{B}}=:(\operatorname{curl} \mathbf{B}) \cdot d \mathbf{S}
\end{gathered}
$$

These definitions guarantee the validity of "standard" integral formulas like

$$
\begin{gathered}
\oint_{\partial \mathcal{D}} \mathbf{E} \cdot d \mathbf{S}=\int_{\mathcal{D}}(\operatorname{div} \mathbf{E}) \hat{\omega} \\
\oint_{\partial \mathcal{S}} \mathbf{B} \cdot d \mathbf{r}=\int_{\mathcal{S}}(\operatorname{curl} \mathbf{B}) \cdot d \mathbf{S}
\end{gathered}
$$

as a consequence of the "spatial" Stokes formula (4b.1). Then from (6.7a) - (6.10) we obtain

$$
\begin{gathered}
\operatorname{div} \mathbf{E}+\mathbf{y} \cdot \mathbf{B}=4 \pi \rho \\
(\operatorname{curl} \mathbf{B}) . d \mathbf{S}-\mathcal{L}_{V}(\mathbf{E} . d \mathbf{S})-(\mathbf{a} \times \mathbf{B}) . d \mathbf{S}=4 \pi \mathbf{j} . d \mathbf{S}
\end{gathered}
$$




$$
\begin{gathered}
(\operatorname{curl} \mathbf{E}) \cdot d \mathbf{S}+\mathcal{L}_{V}(\mathbf{B} \cdot d \mathbf{S})-(\mathbf{a} \times \mathbf{E}) \cdot d \mathbf{S}=0 \\
\operatorname{div} \mathbf{B}-\mathbf{y} \cdot \mathbf{E}=0
\end{gathered}
$$

and

$$
\mathcal{L}_{V}(\rho \hat{\omega})+(\operatorname{div} \mathbf{j}) \hat{\omega}-(\mathbf{a} \cdot \mathbf{j}) \hat{\omega}=0
$$

or (cf. Appendix E)

$$
V \rho+\theta \rho+\operatorname{div} \mathbf{j}-\mathbf{a} \cdot \mathbf{j}=0
$$

as well as the corresponding integral versions

$$
\begin{gathered}
\oint_{\partial \mathcal{D}} \mathbf{E} \cdot d \mathbf{S}=4 \pi \int_{\mathcal{D}} \rho \hat{\omega} \equiv 4 \pi Q \\
\oint_{\partial \mathcal{S}} \mathbf{B} \cdot d \mathbf{r}-\left.\frac{d}{d \tau}\right|_{0} \int_{\Phi_{\tau}(\mathcal{S})} \mathbf{E} \cdot d \mathbf{S}-\int_{\mathcal{S}}(\mathbf{a} \times \mathbf{B}) \cdot d \mathbf{S}=4 \pi \int_{\mathcal{S}} \mathbf{j} \cdot d \mathbf{S} \\
\oint_{\partial \mathcal{S}} \mathbf{E} \cdot d \mathbf{r}+\left.\frac{d}{d \tau}\right|_{0} \int_{\Phi_{\tau}(\mathcal{S})} \mathbf{B} \cdot d \mathbf{S}-\int_{\mathcal{S}}(\mathbf{a} \times \mathbf{E})=0 \\
\oint_{\partial \mathcal{D}} \mathbf{B} \cdot d \mathbf{S}=0
\end{gathered}
$$

and

$$
\left.\frac{d}{d \tau}\right|_{0} \int_{\Phi_{\tau}(\mathcal{D})} \rho \hat{\omega}+\oint_{\partial \mathcal{D}} \mathbf{j} \cdot d \mathbf{S}-\int_{\mathcal{D}}(\mathbf{a} \cdot \mathbf{j}) \hat{\omega}=0
$$

In the simplest situation, i.e. for irrotational $(\mathbf{y}=0)$, geodesic $(\mathbf{a}=0)$ observer field $V\left(\right.$ then $\left.V=\partial_{t}, \tilde{V}=d t\right)$ we get

$$
\begin{gathered}
\operatorname{div} \mathbf{E}=4 \pi \rho \\
(\operatorname{curl} \mathbf{B}) . d \mathbf{S}-\mathcal{L}_{\partial_{t}}(\mathbf{E} . d \mathbf{S})=4 \pi \mathbf{j} . d \mathbf{S} \\
(\operatorname{curl} \mathbf{E}) . d \mathbf{S}+\mathcal{L}_{\partial_{t}}(\mathbf{B} \cdot d \mathbf{S})=0 \\
\operatorname{div} \mathbf{B}=0
\end{gathered}
$$

and

$$
\partial_{t} \rho+\theta \rho+\operatorname{div} \mathbf{j}=0
$$

\section{Appendix I : The equations $d \alpha=\beta$ and $\delta \alpha=\gamma$ for irrotational observer field}

Let $V$ be an irrotational $(\hat{y}=0)$ observer field. Then (cf. (C.8))

$$
\hat{a}=-\hat{d} \Phi \quad \Phi \equiv \ln \psi \quad \tilde{V}=\psi d t \quad V=\psi^{-1} \partial_{t}
$$

$\left(e^{\Phi} \equiv \psi\right.$ - lapse function, cf. [10]). Let us study the equation of the structure

$$
d \alpha=\beta
$$

If

$$
\alpha \leftrightarrow\left(\begin{array}{c}
\hat{s} \\
\hat{r}
\end{array}\right) \quad \beta \leftrightarrow\left(\begin{array}{c}
\hat{S} \\
\hat{R}
\end{array}\right)
$$

we have

$$
\left(\begin{array}{cc}
-\hat{d}+\hat{a} & \mathcal{L}_{V} \\
0 & \hat{d}
\end{array}\right)\left(\begin{array}{c}
\hat{s} \\
\hat{r}
\end{array}\right)=\left(\begin{array}{c}
\hat{S} \\
\hat{R}
\end{array}\right)
$$

or

$$
(-\hat{d}+\hat{a}) \hat{s}+\mathcal{L}_{V} \hat{r}=\hat{S} \quad \hat{d} \hat{r}=\hat{R}
$$

Now

$$
(-\hat{d}+\hat{a}) \hat{s}=-\hat{d} \hat{s}-\hat{d} \Phi \wedge \hat{s}=-e^{-\Phi} \hat{d}\left(e^{\Phi} \hat{s}\right)
$$




$$
\mathcal{L}_{V} \hat{r}=e^{-\Phi} \mathcal{L}_{\partial_{t}} \hat{r}
$$

so that we obtain

$$
-\hat{d}\left(e^{\Phi} \hat{s}\right)+\mathcal{L}_{\partial_{t}} \hat{r}=e^{\Phi} \hat{S} \quad \hat{d} \hat{r}=\hat{R}
$$

Thus we have the simple rule : the acceleration term $\hat{a}=-\hat{d} \Phi$ manifests itself only through the replacement

$$
\hat{s} \mapsto e^{\Phi} \hat{s} \equiv \psi \hat{s} \quad \hat{S} \mapsto e^{\Phi} \hat{S} \equiv \psi \hat{S}
$$

of the upper components of (I.3) (the lower ones being unchanged) in the corresponding equations with $\hat{a}=0$, i.e. in

$$
-\hat{d} \hat{s}+\mathcal{L}_{\partial_{t}} \hat{r}=\hat{S} \quad \hat{d} \hat{r}=\hat{R}
$$

The similar analysis repeated for the equation

$$
\delta \alpha=\gamma \quad \text { where } \gamma \leftrightarrow\left(\begin{array}{c}
\hat{\mathfrak{S}} \\
\hat{\mathfrak{R}}
\end{array}\right)
$$

shows that the replacement to be performed in the corresponding equations with $\hat{a}=0$ is

$$
\hat{r} \mapsto e^{\Phi} \hat{r} \equiv \psi \hat{r} \quad \hat{\mathfrak{R}} \mapsto e^{\Phi} \hat{\mathfrak{R}} \equiv \psi \hat{\mathfrak{R}}
$$

i.e. only the lower components do change now.

For the case of the Maxwell equations (6.14), (6.8) and the continuity equation (6.9) it results in the replacements

$$
\hat{E} \mapsto e^{\Phi} \hat{E} \equiv \psi \hat{E} \quad \rho \mapsto e^{\Phi} \rho \equiv \psi \rho
$$

in the homogeneous pair ( $\rho$ is, however, trivial since it is not present there),

$$
\hat{B} \mapsto e^{\Phi} \hat{B} \equiv \psi \hat{B} \quad \hat{j} \mapsto e^{\Phi} \hat{j} \equiv \psi \hat{j}
$$

in the inhomogeneous pair and

$$
\hat{j} \mapsto e^{\Phi} \hat{j} \equiv \psi \hat{j}
$$

in the continuity equation, i.e. the equations for $\hat{a}=-\hat{d} \Phi \operatorname{read}(\mathrm{cf}$. [10], pp.18-19 and Appendix H here)

$$
\begin{aligned}
& \hat{d} \hat{*} \hat{E}=4 \pi \rho \hat{\omega} \quad \hat{d} \hat{*}\left(e^{\Phi} \hat{B}\right)-\mathcal{L}_{\partial_{t}} \hat{*} \hat{E}=4 \pi \hat{*}\left(e^{\Phi} \hat{j}\right) \\
& \hat{d}\left(e^{\Phi} \hat{E}\right)+\mathcal{L}_{\partial_{t}} \hat{B}=0 \quad \hat{d} \hat{B}=0
\end{aligned}
$$

and

$$
\mathcal{L}_{\partial_{t}}(\rho \hat{\omega})+\hat{d} \hat{*}\left(e^{\Phi} \hat{j}\right)=0
$$

\section{References}

a) Present address : Department of Theoretical Physics, Comenius University, Mlynská dolina F2, 84215 Bratislava, Slovakia; E-mail: fecko@fmph.uniba.sk

[1] K.S.Thorne, D.A.Macdonald : Electrodynamics in curved spacetime : $3+1$ formulation, Mon. Not. R. astr. Soc. (1982) 198,339-343 + Microfiche

[2] Ch.W.Misner, J.A.Wheeler : Classical Physics as Geometry, Annals of Physics, 2, 525-603 (1957)

[3] J.Baez,J.P.Munian: Gauge Fields, Knots and Gravity, World Scientific, 1994

[4] I.M.Benn,R.W.Tucker: An Introduction to Spinors and Geometry with Applications in Physics, Adam Hilger, Bristol, 1989

[5] A. Trautman : Fiber Bundles, Gauge Fields, and Gravitation, in A.Held : General Relativity and Gravitation, Vol. 1, Plenum Press 1980

[6] G.F.R. Ellis : Relativistic cosmology, Cargèse Lectures in Physics, Vol.6, 1-60, 1973

[7] N.Straumann : General Relativity and Relativistic Astrophysics, Springer - Verlag 1991, p.439

[8] Ch.W.Misner, K.S.Thorne, J.A.Wheeler : Gravitation, W.H.Freeman and Company, Ex.22.6

[9] V.I.Arnold : Mathematical Methods of Classical Mechanics, Benjamin/Cummings Reading MA, 1978

[10] K.S.Thorne, R.H.Price, D.A.Macdonald : Black Holes : The Membrane Paradigm, Yale Univ. Press 1986 